\title{
Low Energy Asymptotics for Schrödinger Operators with Slowly Decreasing Potentials
}

\author{
Shu Nakamura \\ Department of Mathematical Sciences, University of Tokyo, 3-8-1, Komaba, Meguro-ku, \\ Tokyo 153, Japan
}

Received: 12 March 1993

\begin{abstract}
Low energy behavior of Schrödinger operators with potentials which decay slowly at infinity is studied. It is shown that if the potential is positive then the zero energy is very regular and the resolvent is smooth near 0 . This implies rapid local decay for the solutions of the Schrödinger equation. On the other hand, if the potential is negative then the resolvent has discontinuity at zero energy. Thus one cannot expect local decay faster than order $t^{-1}$ as $t \rightarrow \infty$.
\end{abstract}

\section{Introduction}

In this paper we consider the Schrödinger operator

$$
H=H_{0}+V(x)=-\hbar^{2} \Delta+V(x) \quad \text { on } \quad L^{2}\left(\mathbf{R}^{d}\right), \quad d \geqq 1 .
$$

We will assume $V(x) \sim c|x|^{-\rho}$ as $|x| \rightarrow \infty$, and study the behavior of $(H-z)^{-1}$ near $z=0$. If $\rho>2$ then $V$ is called very short range and the behavior of $(H-z)^{-1}$ near $z=0$ was studied by Jensen, Kato and others (see, e.g., [JK, J, Mu]). If $d=3$ and $\rho$ is sufficiently large, then it is known that $(H-z)^{-1}$ has an asymptotic expansion in $z^{1 / 2}$ :

$$
(H-z)^{-1} \sim B_{-2} z^{-1}+B_{-1} z^{-1 / 2}+B_{0} z^{0}+\cdots, \quad z \rightarrow 0 .
$$

The top term $B_{-2}$ comes from the 0-energy eigenvalue, and $B_{-1}$ comes from the 0 -energy resonance. Since they are unstable under small perturbations, $(H-z)^{-1}$ is generically regular near $z=0$.

On the other hand, if $0<\rho<2$, then $V$ is called slowly decreasing, and it is known that $(H-z)^{-1}$ behaves quite differently near $z=0$. For the one-dimensional case, this problem was studied by Yafaev [Y1] in detail using integral equation techniques. For the higher dimensional case, Yafaev also studied Schrödinger operators with positive slowly decreasing potentials ([Y2]). In particular, he proved that $(1+|x|)^{-\alpha} H^{-m}(1+|x|)^{-\beta}$ is bounded in $L^{2}\left(\mathbf{R}^{d}\right)$ if $\alpha+\beta>m \rho$. Using this estimate, the low energy asymptotics of $(H-z)^{-1}$ was 
studied. The aim of this paper is to prove some a priori estimates that were assumptions in Yafaev's paper, and to generalize his results.

At first we consider the positive case. Here we fix the Planck constant $\hbar=1$, and suppose $0<\rho<2$.

Assumption $(A: \rho)$. (i) $V$ is a smooth real-valued function on $\mathbf{R}^{d}$ such that

$$
\left|\left(\frac{\partial}{\partial x}\right)^{\alpha} V(x)\right| \leqq C_{\alpha}\langle x\rangle^{-\rho-|\alpha|}, \quad x \in \mathbf{R}^{d},
$$

for any multi-index $\alpha$.

(ii) There is $\delta>0$ such that $V(x) \geqq \delta\langle x\rangle^{-\rho}$ for $x \in \mathbf{R}^{d}$.

(iii) There are $\varepsilon>0$ and $R>0$ such that

$$
x \cdot \frac{\partial V}{\partial x}(x) \leqq-\varepsilon|x|^{-\rho} \text { for }|x|>R .
$$

Here we have used the notation $\langle x\rangle=\left(1+|x|^{2}\right)^{1 / 2}$ for $x \in \mathbf{R}^{d} . F(*)$ denotes the characteristic function (or the characteristic function of an operator) designated by (*).

Theorem 1.1. Suppose $(A: \rho)$ with $0<\rho<2$. Then there exist $\beta, \gamma>0$ and $C>0$ such that

$$
\left\|F\left(|x| \leqq \beta \lambda^{-1 / \rho}\right) F(H \leqq \lambda)\right\| \leqq C \exp \left(-\gamma \lambda^{-(1 / \rho-1 / 2)}\right), \quad \text { for } \quad \lambda \in(0,1] .
$$

Remark. Part (i) and (ii) of Assumption $(\mathrm{A}: \rho)$ is not necessary for Theorem 1.1. It holds if $V$ is bounded and satisfies $(\mathrm{A}: \rho)$-(iii).

This result can be considered as an asymptotic estimate on the local density of states, analogous to the Lifshitz tail for random Schrödinger operators. As a direct consequence of Theorem 1.1, we obtain rapid local decay for the semigroup $e^{-t H}, t \geqq 0$ :

Corollary 1.2. There exist $\beta, \gamma>0$ and $C>0$ such that

$$
\left\|F\left(|x| \leqq \beta t^{2 /(\rho+2)}\right) e^{-t H}\right\| \leqq C \exp \left(-\gamma t^{(2-\rho) /(2+\rho)}\right), \quad t>0
$$

Proof. It suffices to show (1.2) for $t>1$. Setting $\lambda=t^{-2 \rho /(\rho+2)}$, we decompose the left-hand side of (1.2) as follows:

$$
\begin{aligned}
& \left\|F\left(|x| \leqq \beta t^{2 /(\rho+2)}\right) e^{-t H}\right\| \\
& \quad \leqq\left\|F\left(|x| \leqq \beta t^{2 /(\rho+2)}\right) F(H \leqq \lambda)\right\|+\left\|F(H>\lambda) e^{-t H}\right\| \\
& \quad=\left\|F\left(|x| \leqq \beta \lambda^{-1 / \rho}\right) F(H \leqq \lambda)\right\|+\left\|F(H>\lambda) e^{-t H}\right\| \\
& \quad \leqq C \exp \left(-\gamma \lambda^{-(1 / \rho-1 / 2)}\right)+e^{-t \lambda} \\
& \quad=C \exp \left(-\gamma t^{(2-\rho) /(2+\rho)}\right)+\exp \left(-t^{(2-\rho) /(2+\rho)}\right), \quad t>1 .
\end{aligned}
$$

Replacing $\gamma$ by $\min (\gamma, 1)$ if necessary, we obtain (1.2).

Next we consider the boundary values of the resolvent. It is well-known that under our assumption,

$$
\langle x\rangle^{-\gamma}(H-\lambda \pm i 0)^{-1}\langle x\rangle^{-\gamma} \equiv \lim _{\varepsilon \downarrow 0}\langle x\rangle^{-\gamma}(H-\lambda \pm i \varepsilon)^{-1}\langle x\rangle^{-\gamma} \in B\left(L^{2}\left(\mathbf{R}^{d}\right)\right)
$$

exist for $\gamma>1 / 2$ and $\lambda>0$. 
Theorem 1.3. Suppose $V$ satisfies $(A: \rho)$ with $0<\rho<2$ and let $\gamma>1 / 2+\rho / 4$. Then

$$
\sup _{0<\lambda \leqq 1}\left\|\langle x\rangle^{-\gamma}(H-\lambda \pm i 0)^{-1}\langle x\rangle^{-\gamma}\right\| \leqq C<\infty .
$$

Moreover, $\langle x\rangle^{-\gamma}(H-\lambda \pm i 0)^{-1}\langle x\rangle^{-\gamma}$ is Hölder continuous in $\lambda$ near $\lambda=0$.

We can prove similar estimates for powers of the resolvent:

Theorem 1.4. Suppose $V$ satisfies $(A: \rho)$ with $0<\rho<2$. Let $k \geqq 1$ and let $\gamma>\max (k-1 / 2, k(1 / 2+\rho / 4))$. Then

$$
\sup _{0<\lambda \leqq 1}\left\|\langle x\rangle^{-\gamma}(H-\lambda \pm i 0)^{-k}\langle x\rangle^{-\gamma}\right\| \leqq C_{k}<\infty .
$$

Moreover, $\langle x\rangle^{-\gamma}(H-\lambda \pm i 0)^{-k}\langle x\rangle^{-\gamma}$ is Hölder continuous in $\lambda$ near $\lambda=0$.

Since (at least formally),

$$
\left(\frac{d}{d \lambda}\right)^{k}(H-\lambda \pm i 0)^{-1}=k !(H-\lambda \pm i 0)^{-k-1},
$$

Theorem 1.4 implies differentiability of $(H-\lambda \pm i 0)^{-1}$ in $\lambda$ :

Corollary 1.5. Let $\varphi$ be a rapidly decreasing function on $\mathbf{R}^{d}$. Then $\varphi(H-\lambda \pm i 0)^{-1} \varphi$ is $C^{\infty}$-smooth with respect to $\lambda\left(\right.$ in $B\left(L^{2}\left(\mathbf{R}^{d}\right)\right)$-topology).

We denote the spectral projection of $H$ by $E(\lambda)=F(H \leqq \lambda)$. Since

$$
E^{\prime}(\lambda)=(2 \pi i)^{-1}\left((H-\lambda-i 0)^{-1}-(H-\lambda+i 0)^{-1}\right),
$$

we can obtain corresponding estimates for $E^{\prime}(\lambda)$ from Theorems 1.4 and 1.5. In fact, we can prove slightly stronger estimates for $E^{\prime}(\lambda)$ and Theorem 1.4 follow from them:

Theorem 1.6. Suppose $V$ satisfies $(A: \rho)$ with $0<\rho<2$. Let $k \geqq 1$ and let $\gamma>k-1 /$ 2. Then

$$
\left\|\langle x\rangle^{-\gamma}\left(\frac{d}{d \lambda}\right)^{k-1} E^{\prime}(\lambda)\langle x\rangle^{-\gamma}\right\| \leqq C_{k} \lambda^{\delta}, \quad \lambda \in(0,1],
$$

where $\delta=2 \gamma / \rho-k(1 / \rho+1 / 2)$.

Combining these results with the method of Jensen, Mourre and Perry [JMP], we obtain the following uniform decay estimates for the Schrödinger timeevolution:

Theorem 1.7. Suppose $(A: \rho)$ with $0<\rho<2$. Then for any $\gamma>\beta>0$,

$$
\left\|\langle x\rangle^{-\gamma} e^{-i t H}\langle x\rangle^{-\gamma}\right\| \leqq C\langle t\rangle^{-\beta}, \quad t \in \mathbf{R} .
$$

Remark. For the free case, i.e., if $V(x)=0$, then (1.6) holds only if $\beta \leqq d / 2$. Thus a particle in a potential satisfying $(\mathrm{A}: \rho)$ escapes from a finite region faster than a free particle.

We can also estimate $\langle x\rangle^{-\gamma}(H-z)^{-1}\langle x\rangle^{-\gamma}$ in a neighborhood of $z=0$ :

Theorem 1.8. Suppose $(A: \rho)$ with $0<\rho<2$ and let $\gamma>1 / 2+\rho / 4$. Then $\langle x\rangle^{-\gamma}(H-z)^{-1}\langle x\rangle^{-\gamma}$ is uniformly bounded in $z \in \mathbf{C} \backslash[0, \infty)$. 
The idea of the proof of Theorems 1.1-1.8 is as follows: If we change the coordinate: $x=\lambda^{-1 / \rho} y$, then $H$ has the form

$$
H=\lambda\left(-g^{2} \Delta_{y}+V_{\lambda}(y)\right) \equiv \lambda H_{\lambda},
$$

where $g=\lambda^{(1 / \rho-1 / 2)}$ and $V_{\lambda}(y)=\lambda^{-1} V\left(\lambda^{-1 / \rho} y\right)$. Since $\rho<2, g \downarrow 0$ as $\lambda \downarrow 0$, and $(\mathrm{A}: \rho)$ implies

$$
\delta\langle y\rangle^{-\rho} \leqq V_{\lambda}(y) \leqq C|y|^{-\rho}, \quad y \in \mathbf{R}^{d} .
$$

Thus $H_{\lambda}$ has the form of a semiclassical Hamiltonian, and we can apply the methods of semiclassical analysis. Then, for example, Theorem 1.1 follows from the Agmon estimate for a classically forbidden region, and Theorem 1.3 follows from the semiclassical resolvent estimates (see, e.g., [S, HS, RT, GM, HN], etc.). The proof is discussed in Sect. 2.

Next we consider the negative case, i.e., $V(x)<0$. Then the situation is quite different from the positive case. Zero is always the accumulation point of negative eigenvalues, and hence zero seems to be very singular. In order to make the problem manageable, we suppose the Planck constant $\hbar$ is sufficiently small. Note that this is equivalent to replace $V$ by $\mu V$ with a large coupling constant $\mu$.

Assumption $(B: \rho)$. (i) $V$ is smooth real-valued function on $\mathbf{R}^{d}$ such that

$$
\left|\left(\frac{\partial}{\partial x}\right)^{\alpha} V(x)\right| \leqq C_{\alpha}\langle x\rangle^{-\rho-|\alpha|}, \quad x \in \mathbf{R}^{d}
$$

for any multi-index $\alpha$.

(ii) There is $\delta>0$ such that $V(x) \leqq-\delta\langle x\rangle^{-\rho}$ for $x \in \mathbf{R}^{d}$.

$$
\sup _{x \in \mathbf{R}^{d}}|V(x)|^{-1}\left|x \cdot \frac{\partial V}{\partial x}(x)\right| \equiv \rho^{\prime}<2 .
$$

Theorem 1.9. Suppose $V$ satisfies $(B: \rho)$ with $0<\rho<2$. Then there exists $\hbar_{0}>0$ such that if $0<\hbar<\hbar_{0}$ and $\gamma>1 / 2+\rho / 4$ then

$$
\sup _{0<\lambda \leqq 1}\left\|\langle x\rangle^{-\gamma}(H-\lambda \pm i 0)^{-1}\langle x\rangle^{-\gamma}\right\| \leqq C<\infty \text {. }
$$

Moreover,

$$
\langle x\rangle^{-\gamma}(H-0 \pm i 0)^{-1}\langle x\rangle^{-\gamma} \equiv \lim _{\lambda \downarrow 0}\langle x\rangle^{-\gamma}(H-\lambda \pm i 0)^{-1}\langle x\rangle^{-\gamma} \in B\left(L^{2}\left(\mathbf{R}^{d}\right)\right)
$$

exist.

Remark. (i) The above result implies the existence of

$$
\langle x\rangle^{-\gamma} E^{\prime}(+0)\langle x\rangle^{-\gamma} \equiv \lim _{\lambda \downarrow 0}\langle x\rangle^{-\gamma} E^{\prime}(\lambda)\langle x\rangle^{-\gamma}
$$

for $\gamma>1 / 2+\rho / 4$. In general $E^{\prime}(+0) \neq 0$ (see Yafaev [Y1] for one-dimensional case). Thus we may expect at most the decay of order $O\left(t^{-1}\right)$ for $\left\|\langle x\rangle^{-\gamma} e^{-i t H}\langle x\rangle^{-\gamma}\right\|$ even if $\gamma$ is very large.

(ii) Since zero is the accumulation point of $\sigma_{p}(H),(1.8)$ might look like a contradiction. But it is not, since we have additional weight $\langle x\rangle^{-\gamma}$. Instead, if $H \psi_{n}=\lambda_{n} \psi_{n},\left\|\psi_{n}\right\|=1, \lambda_{n} \uparrow 0$, then Theorem 1.9 implies $\left\|\langle x\rangle^{\gamma} \psi_{n}\right\| \geqq C\left|\lambda_{n}\right|^{-1 / 2}$ 
The idea of the proof of Theorem 1.9 is completely different from the positive case though we also use the semiclassical method. We consider a second-order elliptic operator $L_{0}=(-V)^{-1 / 2} H_{0}(-V)^{-1 / 2}$. As we shall see later, we can construct scattering theory for $L_{0}$, and formally we have

$$
(H-0 \pm i 0)^{-1}=\left(H_{0}-(-V) \pm i 0\right)^{-1}=(-V)^{-1 / 2}\left(L_{0}-1 \pm i 0\right)^{-1}(-V)^{-1 / 2} .
$$

Thus $(H-0 \pm i 0)^{-1}$ can be represented by the boundary values of resolvent of $L_{0}$ at energy 1 . This argument is justified in Sect. 3 and Theorem 1.9 follows.

\section{Positive Potentials}

Throughout this section we suppose $V$ satisfies Assumption $(\mathrm{A}: \rho)$ with $0<\rho<2$, and we let $\hbar=1$.

Let $\lambda>0$ be an energy and we change the coordinates:

$$
x \in \mathbf{R}^{d} \rightarrow y \in \mathbf{R}^{d}, \quad x=\lambda^{-1 / \rho} y .
$$

Then the Hamiltonian is transformed to

$$
\begin{aligned}
H & =-\Delta_{x}+V(x) \\
& =\lambda\left\{-\lambda^{2(1 / \rho-1 / 2)} \Delta_{y}+\lambda^{-1} V\left(\lambda^{-1 / \rho} y\right)\right\} \\
& =\lambda\left\{-g^{2} \Delta_{y}+V_{\lambda}(y)\right\}=\lambda H_{\lambda},
\end{aligned}
$$

where $g=\lambda^{1 / \rho-1 / 2}$ and $V_{\lambda}(y)=\lambda^{-1} V\left(\lambda^{-1 / \rho} y\right)$. Since $0<\rho<2, g \downarrow 0$ as $\lambda \downarrow 0$, and we can consider $g$ as a semiclassical parameter. By $(\mathrm{A}: \rho), V_{\lambda}$ satisfies

$$
\begin{aligned}
\left|\left(\frac{\partial}{\partial y}\right)^{\alpha} V_{\lambda}(y)\right| & \leqq C_{\alpha} \lambda^{-1-|\alpha| / \rho}\left\langle\lambda^{-1 / \rho} y\right\rangle^{-\rho-|\alpha|} \\
& \leqq C_{\alpha} \min \left(\lambda^{-1-|\alpha| / \rho},|y|^{-\rho-|\alpha|}\right), \quad y \in \mathbf{R}^{d}, \\
V_{\lambda}(y) & \geqq \delta \lambda^{-1}\left\langle\lambda^{-1 / \rho} y\right\rangle^{-\rho} \geqq \delta\left(\lambda^{1 / \rho}+|y|\right)^{-\rho}, \quad y \in \mathbf{R}^{d} .
\end{aligned}
$$

Theorem 1.1 follows from the above scaling and an Agmon-type semiclassical estimate for the classically forbidden region.

Proof of Theorem 1.1. By the scaling, it suffices to show

$$
\left\|F(|y| \leqq \beta) F\left(H_{\lambda} \leqq 1\right)\right\| \leqq C \exp \left(-\gamma g^{-1}\right), \quad \lambda>0,
$$

for some $\beta, \gamma>0$. We take $\lambda_{0}, \beta>0$ so small that $\delta\left(\lambda_{0}^{1 / \rho}+2 \beta\right)^{-\rho}>2$. Thus if $0<\lambda \leqq \lambda_{0}$ and $|y| \leqq 2 \beta$, then $V_{\lambda}>2$. In other words, $\{y|| y \mid \leqq 2 \beta\}$ is in the forbidden region for a classical particle with energy less than 2 . Hence by Theorem III-1 of [BCD] we obtain

$$
\begin{aligned}
& \|F(|y| \leqq \beta) F(H \leqq 1)\| \\
& \quad \leqq C\left(\sup _{y}\left|V_{\lambda}(y)\right|^{1 / 2}\right) \exp \left(-g^{-1} \int_{\beta}^{2 \beta}\left[\delta\left(\lambda^{1 / \rho}+\mu\right)^{-\rho}-3 / 2\right]^{1 / 2} d \mu\right) \\
& \quad \leqq C \lambda^{-1 / 2} \exp \left(-2 \gamma g^{-1}\right) \leqq C \exp \left(-\gamma g^{-1}\right)
\end{aligned}
$$

with some $\gamma, C>0$ if $g$ is sufficiently small. 
In order to obtain estimates for the boundary values of resolvent, we first note that we can prove an analogue of the semiclassical resolvent estimates for $H_{\lambda}$ (cf. [RT, GM, HN, W, N2], etc.).

Lemma 2.1. Let $k \geqq 1$ and let $\gamma>k-1 / 2$. Then

$$
\left\|\langle y\rangle^{-\gamma}\left(H_{\lambda}-1 \pm i 0\right)^{-k}\langle y\rangle^{-\gamma}\right\| \leqq C_{k} g^{-k}, \quad \lambda \in(0,1]
$$

where $g=\lambda^{1 / \rho-1 / 2}$.

Proof. Let $\lambda_{0}$ and $\beta$ be as in the proof of Theorem 1.1, and let $0<\lambda \leqq \lambda_{0}$, $R>\lambda^{-1 / \rho} \beta$, i.e., $\lambda<(\beta / R)^{\rho}$, where $R$ is the constant in (A: $\left.\rho\right)$-(iii). By the assumption, there exists $\varepsilon>0$ such that

$$
\left(V_{\lambda}(y)-1\right)+\frac{1}{2} y \cdot \frac{\partial V_{\lambda}}{\partial y}(y) \leqq-\varepsilon \quad \text { if } V_{\lambda}(y) \leqq 1+\varepsilon,
$$

uniformly for small $\lambda$. Equation (2.6) implies that the classical particle in the potential $V_{\lambda}$ is nontrapping at energy 1 . Then we follow the argument in [HN]. By virtue of (2.2) and (2.3), all the estimates are uniform for small $\lambda$, and we obtain (2.5) with $k=1$. Combining the Mourre estimate in the above proof with the method of Jensen, Mourre and Perry [PSS] (see [W] and [N2] for the semiclassical form), we obtain (2.5) for $k \geqq 2$.

Lemma 2.2. Let $\beta$ be sufficiently small and let $f \in C_{0}^{\infty}(1 / 2,3 / 2)$. Then for any $N$ and $M$, there is $C>0$ such that

$$
\left\|\langle y\rangle^{M} f\left(H_{\lambda}\right) F(|y| \leqq \beta)\right\| \leqq C \lambda^{N}, \quad \lambda \in(0,1] .
$$

Proof. Instead of $\langle y\rangle$ we use the following weight function $\Lambda \in C^{\infty}\left(\mathbf{R}^{d}\right)$ such that $\Lambda(y)=\Lambda(|y|)$ is nondecreasing in $|y|$ and

$$
\Lambda(y)=\left\{\begin{array}{cc}
1 & \text { if }|y| \leqq 1 \\
|y| & \text { if }|y| \geqq 2
\end{array}\right.
$$

We start by considering $\Lambda f\left(H_{\lambda}\right) F(|y| \leqq \beta)$, where $\beta$ is chosen as in the Proof of Theorem 1.1,

$$
\Lambda f\left(H_{\lambda}\right) F(|y| \leqq \beta)=f\left(H_{\lambda}\right) \Lambda F(|y| \leqq \beta)+\left[\Lambda, f\left(H_{\lambda}\right)\right] F(|y| \leqq \beta) .
$$

The first term in the right-hand side is of order $g^{N}$ for any $N$ as in the proof of (2.4). Thus it suffices to estimate the other term. We set

$$
\operatorname{ad}_{B}^{0}(A)=A, \quad \operatorname{ad}_{B}^{k}(A)=\left[B, \operatorname{ad}_{B}^{k-1}(A)\right] \text { for } k \geqq 1 .
$$

Then it is known that for any $m \geqq 1$,

$$
\begin{aligned}
{[A, f(B)]=} & -\sum_{k=1}^{m-1} \frac{(-1)^{k}}{k !} \operatorname{ad}_{B}^{k}(A) f^{(k)}(B) \\
& +\frac{(-1)^{m}}{2 \pi i} \int_{\mathbf{C}} \partial_{\bar{z}} \tilde{f}(z)(B-z)^{-1} \operatorname{ad}_{B}^{m}(A)(B-z)^{-m} d z d \bar{z}
\end{aligned}
$$


where $\tilde{f}$ is an almost analytic continuation of $f$ (see, e.g., [G], Appendix). Under our assumption,

$$
\operatorname{ad}_{H_{\lambda}}^{k}(\Lambda)=\sum_{j=0}^{k} a_{k j}(\lambda, y)\left(g \frac{\partial}{\partial y}\right)^{j}, \quad k \geqq 1,
$$

where $a_{k j}$ are smooth in $y$, supported away from $\{y|| y \mid<1\}$ and satisfy

$$
\left|\left(\frac{\partial}{\partial y}\right)^{\alpha} a_{k j}(\lambda, y)\right| \leqq C_{k j \alpha} g^{k}\langle y\rangle^{-(k-1)-|\alpha|}, \quad y \in \mathbf{R}^{d}, \quad \lambda \in(0,1] .
$$

From these, we learn

$$
\left\|\operatorname{ad}_{H_{\lambda}}^{k}(\Lambda)\left(H_{\lambda}+1\right)^{-k}\right\| \leqq C_{k} g^{k}, \quad \lambda \in(0,1] .
$$

On the other hand, $\tilde{f}$ is compactly supported and satisfying $\left|\partial_{\bar{z}} \tilde{f}(z)\right| \leqq C_{m}|\operatorname{Im} z|^{m}$ for any $m$. Hence

$$
\left\|\int_{\mathrm{C}} \partial_{\bar{z}} \tilde{f}(z)(H-z)^{-1} \mathrm{ad}_{H_{\lambda}}^{m}(\Lambda)\left(H_{\lambda}-z\right)^{-m} d z d \bar{z}\right\| \leqq C_{m} g^{m} .
$$

Then we use the commutator formula to obtain

$$
\begin{aligned}
& \left\|\left[\Lambda, f\left(H_{\lambda}\right)\right] F(|y| \leqq \beta)\right\| \\
& \leqq \sum_{k=1}^{m} \frac{1}{k !}\left\|\operatorname{ad}_{H_{\lambda}}^{k}(\Lambda)\left(H_{\lambda}+1\right)^{-k}\right\| \cdot\left\|\left(H_{\lambda}+1\right)^{k} f^{(k)}\left(H_{\lambda}\right) F(|y| \leqq \beta)\right\| \\
& \quad+\left\|\int_{\mathrm{C}} \partial_{\bar{z}} \tilde{f}(z)(H-z)^{-1} \operatorname{ad}_{H_{\lambda}}^{m}(\Lambda)\left(H_{\lambda}-z\right)^{-m} d z d \bar{z}\right\| \\
& \leqq C_{m} g^{m}
\end{aligned}
$$

for any $m \geqq 1$. Here we have used an analogue of (2.4) to estimate the term: $\left\|(H+1)^{k} f^{(\bar{k})}\left(H_{\lambda}\right) F(|y| \leqq \beta)\right\|$. Since $m$ is arbitrary, this proves (2.7) with $M=1$. Repeating this procedure for multiple commutators: $\left[\Lambda,\left[\Lambda, f\left(H_{\lambda}\right)\right]\right]$, etc., we obtain (2.7) for any $M$. We omit the detail.

Lemma 2.3. If $f \in C_{0}^{\infty}(1 / 2,3 / 2), k \geqq 1$ and $\gamma>k-1 / 2$, then

$$
\left\|\langle x\rangle^{-\gamma}(H-\lambda \pm i 0)^{-k} f(H / \lambda)\langle x\rangle^{-\gamma}\right\| \leqq C \lambda^{a(k, \gamma)}, \quad \lambda \in(0,1],
$$

where $a(k, \gamma)=2 \gamma / \rho-k(1 / \rho+1 / 2)$.

Proof. It suffices to show (2.8) for small $\lambda$. We show

$$
\left\|\left(\lambda^{1 / \rho}+|y|\right)^{-\gamma}\left(H_{\lambda}-1 \pm i 0\right)^{-k} f\left(H_{\lambda}\right)\left(\lambda^{1 / \rho}+|y|\right)^{-\gamma}\right\| \leqq C g^{-k} .
$$

By Lemma 2.1 we have

$$
\begin{aligned}
& \|\left(\lambda^{1 / \rho}+|y|\right)^{-\gamma} F(|y| \geqq \beta)\left(H_{\lambda}-1 \pm i 0\right)^{-k} f\left(H_{\lambda}\right) \\
& \times F(|y| \geqq \beta)\left(\lambda^{1 / \rho}+|y|\right)^{-\gamma} \| \leqq C g^{-k},
\end{aligned}
$$


where $\beta$ is chosen so small as in Lemma 2.2. On the other hand we also have

$$
\begin{aligned}
& \|\left(\lambda^{1 / \rho}+|y|\right)^{-\gamma} F(|y|<\beta)\left(H_{\lambda}-1 \pm i 0\right)^{-k} f\left(H_{\lambda}\right) \\
& \times F(|y| \geqq \beta)\left(\lambda^{1 / \rho}+|y|\right)^{-\gamma} \| \\
\leqq & C \lambda^{-\gamma / \rho}\left\|F(|y|<\beta) f\left(H_{\lambda}\right)\langle y\rangle^{\gamma}\right\|\left\|\langle y\rangle^{-\gamma}\left(H_{\lambda}-1 \pm i 0\right)^{-k}\langle y\rangle^{-\gamma}\right\| \\
\leqq & C_{m} g^{m}
\end{aligned}
$$

for any $m \geqq 0$. Similarly we can show

$$
\begin{aligned}
& \|\left(\lambda^{1 / \rho}+|y|\right)^{-\gamma} F(|y|<\beta)\left(H_{\lambda}-1 \pm i 0\right)^{-k} f\left(H_{\lambda}\right) \\
& \quad \times F(|y|<\beta)\left(\lambda^{1 / \rho}+|y|\right)^{-\gamma} \| \leqq C_{m} g^{m},
\end{aligned}
$$

for any $m \geqq 0$. Combining them we obtain (2.9).

Now we change the coordinates to obtain estimates for operators in $x$-space. Then we have

$$
\begin{aligned}
&\left\|(1+|x|)^{-\gamma}(H-\lambda \pm i 0)^{-k} f(H / \lambda)(1+|x|)^{-\gamma}\right\| \\
&= \|\left\{\lambda^{-1 / \rho}\left(\lambda^{1 / \rho}+|y|\right)\right\}^{-\gamma} \lambda^{-k}\left(H_{\lambda}-1 \pm i 0\right)^{-k} f\left(H_{\lambda}\right) \\
& \times\left\{\lambda^{-1 / \rho}\left(\lambda^{1 / \rho}+|y|\right)\right\}^{-\gamma} \| \\
& \leqq C \lambda^{-k} \lambda^{2 \gamma / \rho} g^{-k}=C \lambda^{a(k, \gamma) .}
\end{aligned}
$$

As a direct consequence of Lemma 2.3 we obtain Theorem 1.6 since $f(H /$ 2) $E^{\prime}(\lambda)=E^{\prime}(\lambda)$ if $f(1)=1$. Thus if $\gamma>k-1 / 2$ and $\gamma \geqq k(1 / 2+\rho / 4)$ then we observe that $\langle x\rangle^{-\gamma}(d / d \lambda)^{k-1} E^{\prime}(\lambda)\langle x\rangle^{-\gamma}$ is bounded for $\lambda \in(0,1]$. Moreover, using complex interpolation with respect to $\gamma$, we learn that if $\gamma>k(1 / 2+\rho / 4)$ then $\langle x\rangle^{-\gamma}(d / d \lambda)^{k-1} E^{\prime}(\lambda)\langle x\rangle^{-\gamma}$ is Hölder continuous. Now we note that

$$
\sup _{0<\varepsilon \leqq 1}\left|\int_{-1}^{1} \frac{\varphi(x)}{x \pm i \varepsilon} d x\right| \leqq C_{\alpha}\|\varphi\|_{C^{\alpha}}, \quad \varphi \in C^{\alpha}(\mathbf{R}), \quad \alpha>0,
$$

where $C^{\alpha}(\mathbf{R})$ denotes the Hölder space of order $\alpha$. Then, since

$$
(H-z)^{-1} F(H \leqq 1)=\int_{0}^{1} E^{\prime}(\lambda)(\lambda-z)^{-1} d \lambda,
$$

Theorem 1.3 and Theorem 1.8 follow from the Hölder continuity of $\langle x\rangle^{-\gamma} E^{\prime}(\lambda)\langle x\rangle^{-\gamma}$. Theorem 1.4 also follows from the Hölder continuity of $\langle x\rangle^{-\gamma}(d / d \lambda)^{k-1} E^{\prime}(\lambda)\langle x\rangle^{-\gamma}$ and by using integration by parts.

At last we prove Theorem 1.7. By mimicking the proof of Theorem 5.1 of [JMP], we conclude from Theorem 1.6 that for any $\gamma>\beta>0$,

$$
\left\|\langle x\rangle^{-\gamma} e^{-i t H} \chi(H)\langle x\rangle^{-\gamma}\right\| \leqq C\langle t\rangle^{-\beta}, \quad t \in \mathbf{R},
$$

where $\chi \in C_{0}^{\infty}(-1,1)$ is 1 in a neighborhood of 0 . On the other hand, it is well-known that for any $\gamma>0$,

$$
\left\|\langle x\rangle^{-\gamma} e^{-i t H} \tilde{\chi}(H)\langle x\rangle^{-\gamma}\right\| \leqq C\langle t\rangle^{-\gamma}, \quad t \in \mathbf{R},
$$

if $\tilde{\chi}$ is smooth, bounded and supported away from 0 (see, e.g., [I]). Theorem 1.7 follows from these. 


\section{Negative Potentials}

In this section we consider Schrödinger operators with Planck constant $\hbar>0$ and we always suppose $V$ satisfies Assumption (B: $\rho$ ) with $0<\rho<2$. Let $\lambda \in[0,1]$ be an energy. We set $W_{\lambda}(x)=(-V(x)+\lambda)^{-1 / 2}, x \in \mathbf{R}^{d}$. Then $W_{\lambda}(x)$ satisfies

$$
\left|\left(\frac{\partial}{\partial x}\right)^{\alpha} W_{\lambda}(x)\right| \leqq C_{\alpha} \min \left(\langle x\rangle^{\rho / 2-|\alpha|}, \lambda^{-1 / 2-|\alpha|}\right), \quad x \in \mathbf{R}^{d}, \quad \lambda \in[0,1)
$$

for any $\alpha$. We consider a second order elliptic operator

$$
L_{\lambda}=W_{\lambda} H_{0} W_{\lambda}=(-V(x)+\lambda)^{-1 / 2}\left(-\hbar^{2} \Delta\right)(-V(x)+\lambda)^{-1 / 2} .
$$

It is easy to see that $L_{\lambda}$ is essentially self-adjoint on $C_{0}^{\infty}\left(\mathbf{R}^{d}\right)$. The dilation generator $A$ is defined by

$$
A=\frac{1}{2 i}\left(x \cdot \frac{\partial}{\partial x}+\frac{\partial}{\partial x} \cdot x\right)
$$

We use the $\hbar$-pseudodifferential operator calculus (see, e.g., [R, N1]). We denote $a \in S(m, g), g=d x^{2} /\langle x\rangle^{2}+d \xi^{2} /\langle\xi\rangle^{2}$, if

$$
\begin{aligned}
& \left|\left(\frac{\partial}{\partial x}\right)^{\alpha}\left(\frac{\partial}{\partial \xi}\right)^{\beta} a(\hbar ; x, \xi)\right| \leqq C_{\alpha \beta} m(\hbar ; x, \xi)\langle x\rangle^{-|\alpha|}\langle\xi\rangle^{-|\beta|}, \\
& x, y \in \mathbf{R}^{d}, \quad 0<\hbar \leqq 1,
\end{aligned}
$$

for any $\alpha$ and $\beta$. The Weyl operator $a(\hbar ; x, \hbar D)$ is defined by

$$
a(\hbar ; x, \hbar D) \varphi(x)=(2 \pi \hbar)^{-d} \int e^{i(x-y) \xi / \hbar} a(\hbar ;(x+y) / 2, \xi) \varphi(y) d y d \xi
$$

for $\varphi \in \mathscr{S}\left(\mathbf{R}^{d}\right)$.

We first prove the limiting absorption principle for $L_{\lambda}$.

Lemma 3.1. There exists $\hbar_{0}>0$ such that if $0<\hbar \leqq \hbar_{0}$ then for any $\gamma>1 / 2$,

$$
\sup _{0<\varepsilon \leqq 1}\left\|\langle A\rangle^{-\gamma}\left(L_{\lambda}-1 \pm i \varepsilon\right)^{-1}\langle A\rangle^{-\gamma}\right\| \leqq C<\infty, \quad \lambda \in[0,1],
$$

where $C$ depends only on $\hbar, \gamma$ and $V$. Moreover,

$$
\lim _{\varepsilon \downarrow 0}\langle A\rangle^{-\gamma}\left(L_{\lambda}-1 \pm i \varepsilon\right)^{-1}\langle A\rangle^{-\gamma}=\langle A\rangle^{-\gamma}\left(L_{\lambda}-1 \pm i 0\right)^{-1}\langle A\rangle^{-\gamma}
$$

exist.

Proof. We use the Mourre theory of limiting absorption principle (see, e.g., [Mo, PSS, CFKS]). By direct computations we can show

$$
\left[L_{\lambda}, i A\right]\left(L_{\lambda}+1\right)^{-1},\left[\left[L_{\lambda}, i A\right], i A\right]\left(L_{\lambda}+1\right)^{-1} \in B\left(L^{2}\left(\mathbf{R}^{d}\right)\right) .
$$

Hence it suffices to show the Mourre estimate:

$$
E_{I}\left[L_{\lambda}, i A\right] E_{I} \geqq \delta E_{I}, \quad \delta>0, \quad I=[1 / 2,2] .
$$


Since the principal symbols of $L_{\lambda}$ are $A$ and given by $(-V+\lambda)^{-1} \xi^{2}$ and $\hbar^{-1} x \cdot \xi$, respectively, the principal symbol of $\left[L_{\lambda}, i A\right]$ is given by

$$
\begin{aligned}
\left\{(-V+\lambda)^{-1} \xi^{2}, x \cdot \xi\right\} & =\left(2-\frac{x \cdot \partial V}{-V+\lambda}\right)(-V+\lambda)^{-1} \xi^{2} \\
& \geqq\left(2-\rho^{\prime}\right)(-V+\lambda)^{-1} \xi^{2},
\end{aligned}
$$

where $\{\cdot, \cdot\}$ denotes the Poisson bracket. Moreover, the lower order symbol is in $S\left(\hbar^{2}\langle x\rangle^{\rho-2}, g\right)$. Hence by the Fefferman-Phong inequality we obtain

$$
\left[L_{\lambda}, i A\right] \geqq\left(2-\rho^{\prime}\right) L_{\lambda}-C \hbar^{2}, \quad \hbar \in(0,1],
$$

uniformly in $\lambda \in[0,1]$. If we take $\hbar_{0}$ sufficiently small, (3.4) implies (3.3) with $0<\delta<2-\rho^{\prime}$.

In what follows we fix $\hbar>0$ such that $0<\hbar \leqq \hbar_{0}$.

Lemma 3.2. For each $0<\lambda \leqq 1, \gamma>1 / 2$,

$$
\sup _{0<\varepsilon \leqq 1}\left\|\langle A\rangle^{-\gamma}\left(L_{\lambda}-1 \pm i \varepsilon W_{\lambda}^{2}\right)^{-1}\langle A\rangle^{-\gamma}\right\| \leqq C<\infty \text {. }
$$

Moreover,

$$
\lim _{\varepsilon \downarrow 0}\langle A\rangle^{-\gamma}\left(L_{\lambda}-1 \pm i \varepsilon W_{\lambda}^{2}\right)^{-1}\langle A\rangle^{-\gamma}=\langle A\rangle^{-\gamma}\left(L_{\lambda}-1 \pm i 0\right)^{-1}\langle A\rangle^{-\gamma} .
$$

Remark. The constant $C$ in (3.5) depends on $\lambda$. The right-hand side of (3.6) is defined by (3.2).

Proof. Given the Mourre estimate (3.3), the proof of (3.5) is almost identical with the proof of Lemma 3.1. We only note that we use sup $\left|W_{\lambda}\right|=\lambda^{-1}<\infty$ and $\sup \left|\left[W_{\lambda}^{2}, i A\right]\right| \leqq C \lambda^{-2}<\infty$, and hence the estimates depends on $\lambda$. It remains only to show (3.6). We mimic the proof of the Hölder continuity of $F(z)=\langle A\rangle^{-\gamma}\left(L_{\lambda}-z\right)^{-1}\langle A\rangle^{-\gamma}$ in $z$ (cf. [PSS]). Instead of $\left\|F(z)-F\left(z^{\prime}\right)\right\|$ we estimate $\left\|F(1+i 0)-F\left(1+i 0 \cdot W_{\lambda}^{2}\right)\right\|$. We omit the detail because the modification is obvious and the notations are rather involved.

Lemma 3.3. For any $\beta, \gamma \in \mathbf{R}, \lambda \in[0,1]$,

$$
\langle P\rangle^{\beta+2}\langle x\rangle^{\gamma}\left(L_{\lambda}+1\right)^{-1}\langle x\rangle^{-\gamma}\langle P\rangle^{-\beta} \in B\left(L^{2}\left(\mathbf{R}^{d}\right)\right),
$$

where $P=-i \hbar \partial / \partial x$. Moreover the operator norm is uniformly bounded for $\lambda \epsilon$ $[0,1]$.

Proof. It suffices to show

$$
\langle x\rangle^{\gamma}\left(L_{\lambda}+1\right)^{-1}\langle x\rangle^{-\gamma} \in B\left(L^{2}\left(\mathbf{R}^{d}\right)\right)
$$

and

$$
\langle P\rangle^{\beta+2}\left(L_{\lambda}+1\right)^{-1}\langle P\rangle^{-\beta} \in B\left(L^{2}\left(\mathbf{R}^{d}\right)\right) .
$$


Let $\beta_{0}=1-\rho / 2$ and let $M_{\lambda}=P W_{\lambda}$. Then $L_{\lambda}=M_{\lambda}^{*} M_{\lambda}$ and hence $M_{\lambda}$ is $L_{\lambda}^{1 / 2}$-bounded. By easy computations, $M_{\lambda}^{*}$ is also $L_{\lambda}^{1 / 2}$-bounded. We compute

$$
\begin{aligned}
{\left[\langle x\rangle^{\beta_{0}},\left(L_{\lambda}+1\right)^{-1}\right]=} & \left(L_{\lambda}+1\right)^{-1}\left[L_{\lambda},\langle x\rangle^{\beta_{0}}\right]\left(L_{\lambda}+1\right)^{-1} \\
= & \left(L_{\lambda}+1\right)^{-1}\left[M_{\lambda}^{*}\left\{M_{\lambda},\langle x\rangle^{\beta_{0}}\right]\right. \\
& \left.+\left[M_{\lambda}^{*},\langle x\rangle^{\beta_{0}}\right] M_{\lambda}\right\}\left(L_{\lambda}+1\right)^{-1} .
\end{aligned}
$$

It is easy to see $\left[M_{\lambda},\langle x\rangle^{\beta_{0}}\right] \in B\left(L^{2}\right)$, etc., and hence $\left[\langle x\rangle^{\beta_{0}},\left(L_{\lambda}+1\right)^{-1}\right] \in B\left(L^{2}\right)$. This implies (3.8) with $\gamma=\beta_{0}$. Iterating this procedure we can obtain (3.8) for $\gamma=m \beta_{0}, m \geqq 1$. Then the claim follows by complex interpolation.

On the other hand, using $L_{\lambda}^{1 / 2}$-boundedness of $M_{\lambda}$ again, we have

$$
\begin{aligned}
W_{\lambda}^{2} P^{2}\left(L_{\lambda}+1\right)^{-1} \\
=W_{\lambda} P^{2} W_{\lambda}\left(L_{\lambda}+1\right)^{-1}+\left(W_{\lambda} P\left[W_{\lambda}, P\right]+W_{\lambda}\left[W_{\lambda}, P\right] P\right)\left(L_{\lambda}+1\right)^{-1} \\
=L_{\lambda}\left(L_{\lambda}+1\right)^{-1}+2\left[W_{\lambda}, P\right] M_{\lambda}^{*}\left(L_{\lambda}+1\right)^{-1}+W_{\lambda}\left[P,\left[W_{\lambda}, P\right]\right]\left(L_{\lambda}+1\right)^{-1} \\
\quad \in B\left(L^{2}\left(\mathbf{R}^{d}\right)\right),
\end{aligned}
$$

since $M_{\lambda}^{*}$ is $L_{\lambda}$-bounded and $\left|W_{\lambda}\left[P,\left[W_{\lambda}, P\right]\right]\right| \leqq C\langle x\rangle^{\rho-2}$. This implies (3.9) with $\beta=0$. Noting that $\left[P, M_{\lambda}\right] \in B\left(L^{2}\right),(3.9)$ for general $\beta$ is proved by commutator calculations as in the proof of (3.8).

Lemma 3.4. For any $0 \leqq \gamma \leqq 1$,

$$
\left\|\langle A\rangle^{\gamma}\left(L_{\lambda}+1\right)^{-1}\langle x\rangle^{-\gamma} W_{\lambda}^{\gamma}\right\| \leqq C<\infty, \quad \lambda \in[0,1] .
$$

Proof. We show $\left\|A\left(L_{\lambda}+1\right)^{-1}\langle x\rangle^{-1} W_{\lambda}\right\| \leqq C<\infty$. Then (3.10) holds for $\gamma=1$ and (3.10) with $\gamma=0$ is obvious. Hence (3.10) for $0<\gamma<1$ follows by complex interpolation. The above estimate follows from commutator calculations as in the proof of Lemma 3.3, and the fact $M_{\lambda}=P W_{\lambda}$ is $L_{\lambda}$-bounded.

Lemma 3.5. For $1 / 2<\gamma \leqq 1$,

$$
\sup _{0 \leqq \lambda \leqq 1}\left\|\langle x\rangle^{-\gamma} W_{\lambda}^{\gamma}\left(L_{\lambda}-1 \pm i 0\right)^{-1} W_{\lambda}^{\gamma}\langle x\rangle^{-\gamma}\right\| \leqq C<\infty .
$$

Proof. We use Lemmas 3.1 and 3.4. Then

$$
\begin{aligned}
& \left\|\langle x\rangle^{-\gamma} W_{\lambda}^{\gamma}\left(L_{\lambda}-1 \pm i 0\right)^{-1} W_{\lambda}^{\gamma}\langle x\rangle^{-\gamma}\right\| \\
& =\|\langle x\rangle^{-\gamma} W_{\lambda}^{\gamma}\left\{\left(L_{\lambda}+1\right)^{-1}+2\left(L_{\lambda}+1\right)^{-2}\right. \\
& \left.\quad+4\left(L_{\lambda}+1\right)^{-1}\left(L_{\lambda}-1 \pm i 0\right)^{-1}\left(L_{\lambda}+1\right)^{-1}\right\} W_{\lambda}^{\gamma}\langle x\rangle^{-\gamma} \| \\
& \leqq C+\left\|\langle x\rangle^{-\gamma} W_{\lambda}^{\gamma}\left(L_{\lambda}+1\right)^{-1}\langle A\rangle^{\gamma}\right\|^{2}\left\|\langle A\rangle^{-\gamma}\left(L_{\lambda}-1 \pm i 0\right)^{-1}\langle A\rangle^{-\gamma}\right\| \\
& \leqq \\
& \quad 2 C<\infty . \quad \square
\end{aligned}
$$

Now we can prove the first part of Theorem 1.9.

Lemma 3.6. If $\gamma>1 / 2+\rho / 4$, then

$$
\sup _{0<\lambda \leqq 1}\left\|\langle x\rangle^{-\gamma}(H-\lambda \pm i 0)^{-1}\langle x\rangle^{-\gamma}\right\| \leqq C<\infty .
$$


Proof. We first note that by Lemmas 3.2 and 3.4,

$$
\begin{gathered}
\lim \langle x\rangle^{-\gamma} W_{\lambda}^{\gamma}\left(L_{\lambda}-1 \pm i \varepsilon W_{\lambda}^{2}\right)^{-1} W_{\lambda}^{\gamma}\langle x\rangle^{-\gamma} \\
\quad=\langle x\rangle^{-\gamma} W_{\lambda}^{\gamma}\left(L_{\lambda}-1 \pm i 0\right)^{-1} W_{\lambda}^{\gamma}\langle x\rangle^{-\gamma} .
\end{gathered}
$$

On the other hand, it is easy to see

$$
(H-\lambda \pm i \varepsilon)^{-1}=W_{\lambda}\left(L_{\lambda}-1 \pm i \varepsilon W_{\lambda}^{2}\right)^{-1} W_{\lambda} .
$$

Hence we obtain

$$
\begin{gathered}
\lim _{\varepsilon \downarrow 0}\langle x\rangle^{-\gamma} W_{\lambda}^{-(1-\gamma)}(H-\lambda \pm i \varepsilon)^{-1} W_{\lambda}^{-(1-\gamma)}\langle x\rangle^{-\gamma} \\
=\langle x\rangle^{-\gamma} W_{\lambda}^{\gamma}\left(L_{\lambda}-\lambda \pm i 0\right)^{-1} W_{\lambda}^{\gamma}\langle x\rangle^{-\gamma}
\end{gathered}
$$

Thus by Lemma 3.5,

$$
\sup _{0<\lambda \leqq 1}\left\|\langle x\rangle^{-\gamma} W_{\lambda}^{-(1-\gamma)}(H-\lambda \pm i 0)^{-1} W_{\lambda}^{-(1-\gamma)}\langle x\rangle^{-\gamma}\right\| \leqq C<\infty .
$$

Since $\left|W_{\lambda}(x)\right| \leqq C\langle x\rangle^{\rho / 2}$ for $x \in \mathbf{R}^{d}, \lambda \in[0,1]$, it implies

$$
\sup _{0<\lambda \leqq 1}\left\|\langle x\rangle^{-\gamma^{\prime}}(H-\lambda \pm i 0)^{-1}\langle x\rangle^{-\gamma^{\prime}}\right\| \leqq C<\infty,
$$

with $\gamma^{\prime}=\gamma+(1-\gamma) \rho / 2$. Since $\gamma>1 / 2$ if and only if $\gamma^{\prime}>1 / 2+\rho / 4$, this implies the assertion.

In order to prove the existence of $(H-0 \pm i 0)^{-1}$, we show

$$
\lim \langle x\rangle^{-\gamma}(H-\lambda \pm i 0)^{-1}\langle x\rangle^{-\gamma}=\langle x\rangle^{-\gamma} W_{0}\left(L_{0}-1 \pm i 0\right)^{-1} W_{0}\langle x\rangle^{-\gamma} .
$$
$\lambda \downarrow 0$

Lemma 3.7. As $\lambda \downarrow 0, L_{\lambda} \rightarrow L_{0}$ in the strong resolvent sense. i.e., for any $z \in$ $\mathbf{C} \backslash[0, \infty)$,

$$
s-\lim \left(L_{\lambda}-z\right)^{-1}=\left(L_{0}-z\right)^{-1} .
$$

Proof. It suffices to show

$$
\lim _{\lambda \downarrow 0}\left(L_{\lambda}+1\right)^{-1} \varphi=\left(L_{0}+1\right)^{-1} \varphi
$$

for $\varphi \in \mathscr{S}\left(\mathbf{R}^{d}\right)$ (see [RS, Sect. VIII.7]). We have

$$
\begin{aligned}
\left(L_{\lambda}+1\right)^{-1} \varphi-\left(L_{0}+1\right)^{-1} \varphi=\left(L_{\lambda}+1\right)^{-1}\left(L_{0}-L_{\lambda}\right)\left(L_{0}+1\right)^{-1} \varphi \\
=\left(L_{\lambda}+1\right)^{-1}\left(W_{0} P^{2} W_{0}-W_{\lambda} P^{2} W_{\lambda}\right)\langle P\rangle^{-2}\langle x\rangle^{-2} \\
\quad \times\left(\langle x\rangle^{2}\langle P\rangle^{2}\left(L_{0}+1\right)^{-1}\langle P\rangle^{-2}\langle x\rangle^{-2}\right)\left(\langle x\rangle^{2}\langle P\rangle^{2} \varphi\right),
\end{aligned}
$$

and hence by Lemma 3.3,

$$
\left\|\left(L_{\lambda}+1\right)^{-1} \varphi-\left(L_{0}+1\right)^{-1} \varphi\right\| \leqq C \lambda\left\|\langle x\rangle^{2}\langle P\rangle^{2} \varphi\right\| \rightarrow 0
$$

as $\lambda \downarrow 0$. 
Lemma 3.8. For $\gamma>1 / 2$,

$$
\underset{\lambda \downarrow 0}{s-\lim }\langle x\rangle^{-\gamma}\left(L_{\lambda}-1 \pm i 0\right)^{-1}\langle x\rangle^{-\gamma}=\langle x\rangle^{-\gamma}\left(L_{0}-1 \pm i 0\right)^{-1}\langle x\rangle^{-\gamma} .
$$

Proof. We note that the convergence:

$$
\langle x\rangle^{-\gamma}\left(L_{\lambda}-1 \pm i \varepsilon\right)^{-1}\langle x\rangle^{-\gamma} \rightarrow\langle x\rangle^{-\gamma}\left(L_{\lambda}-1 \pm i 0\right)^{-1}\langle x\rangle^{-\gamma} \quad \text { as } \varepsilon \downarrow 0
$$

is uniform in $\lambda \in[0,1]$ since all the estimates in the Mourre theory is uniform in $\lambda$. Hence Lemma 3.7 implies (3.15).

Proof of Theorem 1.9. Let $\varphi, \psi \in \mathscr{S}\left(\mathbf{R}^{d}\right)$. Then it follows from Lemma 3.8 that if $\gamma>1 / 2+\rho / 4$ and $\beta>1 / 2$,

$$
\begin{aligned}
\left(\varphi,\langle x\rangle^{-\gamma}(H-\lambda \pm i 0)^{-1}\langle x\rangle^{-\gamma} \psi\right) \\
=\left(\varphi,\langle x\rangle^{-\gamma} W_{\lambda}\left(L_{\lambda}-1 \pm i 0\right)^{-1} W_{\lambda}\langle x\rangle^{-\gamma} \psi\right) \\
=\left(\left(W_{\lambda}\langle x\rangle^{\beta-\gamma} \varphi\right),\left[\langle x\rangle^{-\beta}\left(L_{\lambda}-1 \pm i 0\right)^{-1}\langle x\rangle^{-\beta}\right]\left(W_{\lambda}\langle x\rangle^{-\beta-\gamma} \psi\right)\right) \\
\quad \rightarrow\left(\left(W_{0}\langle x\rangle^{\beta-\gamma} \varphi\right),\left[\langle x\rangle^{-\beta}\left(L_{0}-1 \pm i 0\right)^{-1}\langle x\rangle^{-\beta}\right]\left(W_{0}\langle x\rangle^{\beta-\gamma} \psi\right)\right) \\
=\left(\varphi,\langle x\rangle^{-\gamma} W_{0}\left(L_{0}-1 \pm i 0\right)^{-1} W_{0}\langle x\rangle^{-\gamma} \psi\right) .
\end{aligned}
$$

This proves (3.13) in the weak sense. Let $\gamma>\gamma^{\prime}>1 / 2+\rho / 4$. Then

$$
\begin{aligned}
\langle x\rangle^{-\gamma} & (H-\lambda \pm i 0)^{-1}\langle x\rangle^{-\gamma} \\
= & \langle x\rangle^{-\gamma}(H+1)^{-1}\langle x\rangle^{-\gamma}+(1+\lambda)\langle x\rangle^{-\gamma}(H+1)^{-2}\langle x\rangle^{-\gamma} \\
& +(1+\lambda)^{2}\left(\langle x\rangle^{-\gamma}(H+1)^{-1}\langle x\rangle^{\gamma^{\prime}}\right) \\
& \times\left(\langle x\rangle^{-\gamma^{\prime}}(H-\lambda \pm i 0)^{-1}\langle x\rangle^{-\gamma^{\prime}}\right)\left(\langle x\rangle^{\gamma^{\prime}}(H+1)^{-1}\langle x\rangle^{-\gamma}\right) .
\end{aligned}
$$

Noting $\langle x\rangle^{\gamma^{\prime}}(H+1)^{-1}\langle x\rangle^{-\gamma}$ is compact, we learn that the weak convergence of $\langle x\rangle^{-\gamma^{\prime}}(H-\lambda \pm i 0)^{-1}\langle x\rangle^{-\gamma^{\prime}}$ implies the norm convergence of $\langle x\rangle^{-\gamma}(H-\lambda \pm i 0)^{-1}\langle x\rangle^{-\gamma}$. This completes the proof of Theorem 1.9.

Acknowledgement The author wishes to thank Professor A Jensen and Professor A.V. Sobolev for valuable discussions.

\section{References}

[BCD] Briet, Ph, Combes, J.M., Duclos, P.: Spectral stability under tunneling Commun. Math. Phys. 126, 133-156 (1989)

[CFKS] Cycon, H.L., Froese, R.G, Kirsch, W, Simon, B.: Schrödinger Operators with Applications to Quantum Mechanics and Global Geometry Berlin, Heidelberg, New York: Springer, 1987

[G] Gérard, Ch.: Sharp resolvent estimates for $N$-particle systems Duke Math. J. 67, 483-515 (1992)

[GM] Gérard, Ch., Martinez, A: Principe d'absorption limite pour des opérateurs de Schrödinger à longue portée C.R. Acad Sci. Paris 306, 121-123 (1989)

[HN] Hislop, P D, Nakamura, S : Semiclassical resolvent estimates. Ann Inst. H. Poincaré 51, 187-198 (1989)

[HS] Helffer, B, Sjöstrand, J : Multiple wells in the semiclassical limit I. Comm P.D.E. 9, 337-408 (1984)

[I] Isozaki, H.: Decay rates of scattering states for Schrödinger operators J Math. Kyoto Univ 26, 595-603 (1986) 
[J] Jensen, A.: Spectral properties of Schrödinger operators and time-decay of the wave functions Results in $L^{2}\left(\mathbf{R}^{m}\right), m \geqq 5$. Duke Math J. 47, 57-80 (1980); Spectral properties of Schrödinger operators and time-decay of the wave functions. Results in $L^{2}\left(\mathbf{R}^{4}\right)$. J. Math. Anal. Appl. 101, 491-513 (1984)

[JK] Jensen, A., Kato, T.: Spectral properties of Schrödinger operators and time-decay of wave functions. Duke Math J 46, 583-611 (1979)

[JMP] Jensen, A., Mourre, E., Perry, P : Multiple commutator estimates and resolvent smoothness in quantum scattering theory Ann. Inst. H. Poincaré 41, 207-225 (1984)

[Mo] Mourre, E.: Absence of singular continuous spectrum for certain self-adjoint operators. Commun. Math. Phys. 78, 391-408 (1981)

$\mathrm{Mu}$ ] Murata, M: Asymptotic expansions in time for solutions of Schrödinger-type equations J. Funct. Anal 49, 10-56 (1982)

[N1] Nakamura, S.: Shape resonances for distortion analytic Schrödinger operators. Comm P.D.E 14, 1383-1419 (1989)

[N2] Nakamura, S.: Resolvent estimates and time-decay in the semi-classical limit. To appear in Asterisque (1993)

[PSS] Perry, P, Sigal, I M., Simon, B.: Spectral analysis of $N$-body Schrödinger operators Ann. Math. 114, 519-567 (1981)

[R] Robert, D.: Autour de l'approximation semiclassique Basel, Boston: Birkhäuser, 1983

[RS] Reed, M., Simon, B.: Methods of Modern Mathematical Physics. Vol 1. Functional Analysis New York: Academic Press, 1972

[RT] Robert, D., Tamura, H : Semiclassical bounds for resolvents for Schrödinger operators and asymptotics for scattering phases, Comm P.D.E. 9, 1017-1058 (1984); Semiclassical bounds for resolvents and asymptotics for total cross-sections Ann. Inst. H. Poincaré 46, 415-442 (1987); Semiclassical asymptotics for local spectral density and time-delay problems in scattering processes J. Funct Anal. 80, 124-147 (1988); Asymptotic behavior of scattering amplitudes in semi-classical and low energy limits. Ann Inst. Fourier, Grenoble 39, 124-147 (1989)

[S] Simon, B.: Semiclassical analysis of low lying eigenvalues II Tunneling. Ann Math. 120, 89-118 (1984)

[W] Wang, X.P.: Time-decay of scattering solutions and resolvent estimates for semiclassical Schrödinger operators. J. Diff. Eq. 71, 348-395 (1988)

[Y1] Yafaev, D.R.: The low energy scattering for slowly decreasing potentials Commun Math Phys. 85, 117-196 (1982)

[Y2] Yafaev, D.R.: Spectral properties of the Schrödinger operator with a potential having a slow falloff. Funct. Anal and its Appl. (Steklov Math. Inst.) 16, 47-54 (1982) (English translation, 1983)

Communicated by H. Araki 